\title{
BRANCHING INTEGRAL FRACTION IN THE THEORY OF THE SPECTRUM OF QUASIPARTICLES INTERACTING WITH PHONONS
}

\author{
M.V.TKACH \\ Chernivtsi State University, Department of Theoretical Physics, \\ 2 M.Kotsyubynskii St., UA-274012 Chernivtsi, Ukraine
}

Received September 12, 1995

\begin{abstract}
For the system of the quasiparticles interacting with arbitrary number of phonon modes at $T=0 \mathrm{~K}$ on the basis of the Feinman diagram technique the exact representation of the Green function in the form of the infinite branching integral fraction was obtained. It is shown that thus the developed method of the calculation of the Green functions monotypically takes into account virtual many-phonon processes, it gives an opportunity to obtain the spectrum of the system at the arbitrary binding values and in any region of energies and quasiimpulses quickly and effectively. It is established that in the system of dispersionless quasiparticles interacting with many-mode phonons the excited states are convergent infinite series of energy levels.
\end{abstract}

\section{Introduction}

The Green function (GF) method is one of widely spread in the theory of the quasiparticles interacting with phonons. Although the different methods of the GF calculation are known, only for the systems with ordinary Hamiltonians sometimes one can perform the canonical or unitary operator transformation which provides the exact solution of the problem of the system spectrum renormalized due to the interaction [1]. In any general case the GF theory is based on the expand into a range by all possible powers of the interaction energy. It is true for all types [1-3] of the Bogolybov method which uses the equation of motion for the operators as well as for traditional types of the Feinman diagram technique [4,5].

It is well known that the basic mathematical problem of the general theory of GF is the fact that in case of the non-weak interaction when it is necessary to take into account higher orders of the theory, the discrepancies of non-physical nature arise. The reason of their appearance had been clear long ago. In 1961 D.Pains wrote in his paper [6] that the nature "did not agree" with the use of power series expand in the many-body problem.

The GF theory which does not use any power series expand was developed by the author of $[7,8]$ for the system of quasiparticles interacting with one-mode phonons. It was based on the cardinal rebuilding of the ranges of the traditional Feinman diagram technique which provided an opportunity to obtain the exact representation of quasiparticles GF in the form of an infinite continued integral fraction. Similar in its conception another essentially different method of the GF representation in the form of the infinite 
continued integral fraction was developed later in the papers $[9,10]$ for the systems with the same Hamiltonian as in [7].

The effectiveness of the developed method of taking into account virtual many-phonon processes researching the spectrum of quasiparticles tightly bound with phonons in the region of arbitrary values of energies and quasiimpetus was demonstrated in the papers [7,8] and in the next papers [10-13] on the examples of particular systems.

The main purpose of this paper is the generalization of the method of effective accounting of virtual many-phonon processes when calculating quasiparticles GF developed in the paper [7] in application to the systems having an arbitrary number of modes. It will be shown that for such systems the quasiparticles GF has the exact representation in the form of an infinite branching integral fraction (IBIF). The quasiparticle-phonon binding functions in any branches of this object are in the form of the squared module. Therefore such representation does not contain different powers and it is effective at any values of the interaction energy. When the phonon system has only one mode of vibrations, the branching fraction degenerates into continued and the GF complies with the results obtained in paper [7].

In the present paper the energy multiplier more compact than the one in the paper [7] is suggested. It is easily applicable to the calculation of particular systems. The approach developed in this paper is used to analyse a spectrum of dispersionless quasiparticles interacting with many-mode phonons. The high efficiency of this method at the arbitrary binding forces is demonstrated. New peculiarities of such systems spectrum are established.

\section{Representation of quasiparticle GF in the form of IBIF}

The spinless quasiparticles (polarons, excitons, impurities, etc.) interacting with phonons at $T=0 \mathrm{~K}$ are considered. The Hamiltonian of the system in the representation of second quantization is typical [1-6]

$$
H=\sum_{\boldsymbol{k}} \varepsilon_{\boldsymbol{k}} a_{\boldsymbol{k}}^{+} a_{\boldsymbol{k}}+\sum_{\lambda, \boldsymbol{k}, \boldsymbol{q}} \varphi_{\lambda}(\boldsymbol{q}) a_{\boldsymbol{k}+\boldsymbol{q}}^{+} a_{\boldsymbol{k}}\left(b_{\boldsymbol{q} \lambda}+b_{-\boldsymbol{q} \lambda}^{+}\right)+\sum_{\lambda \boldsymbol{q}} \Omega_{\lambda \boldsymbol{q}}\left(b_{\boldsymbol{q} \lambda}^{+} b_{\boldsymbol{q} \lambda}+1 / 2\right) .
$$

Here $\varepsilon_{\boldsymbol{k}}$ - quasiparticles dispersion law, $a_{\boldsymbol{k}}^{+}$and $a_{\boldsymbol{k}}$ - operators satisfying commutative or anticommutative expressions depending on the statistics of quasiparticles (fermions, bozons); $\Omega_{\lambda \boldsymbol{q}}$ - phonons dispersion law of mode $\lambda$ with quasimomentum $\boldsymbol{q} ; b_{\boldsymbol{q} \lambda}^{+}$and $b_{\boldsymbol{q} \lambda}$ - operators of bozone type; $\varphi_{\lambda}(\boldsymbol{q})$ is a quasiparticle-phonon binding function $(\lambda=1,2, \ldots, \tau)$.

To obtain a quasiparticle spectrum renormalized due to the interaction one has to build a Fourier image of the total GF $G\left(\boldsymbol{k}, \omega^{\prime}\right)$. It is defined by Daison equation $(\hbar=1)$

$$
G\left(\boldsymbol{k}, \omega^{\prime}\right)=\left\{\omega^{\prime}-\varepsilon_{\boldsymbol{k}}-M\left(\boldsymbol{k}, \omega^{\prime}\right)\right\}^{-1}
$$

using the total mass operator (MO) as a set of the all irreducible Feinman diagrams. In order to receive the representation of MO in the form of IBIF as in the paper [7] we present all diagrams of the MO not simply in the consistency of an increasing number of tops, but arrange them in accordance with two important peculiarities (figure 1): in the order of the power of the squared module of the interaction function (the Roman numerals above the diagrams) and the maximal number of phonon (dashed) lines passing above one of the quasiparticle (solid) lines in the diagrams of the indicated group. 
The Arabic numeral $(n)$ indicates the maximal number of virtual phonons participating in the interaction processes described by the given class of diagrams.

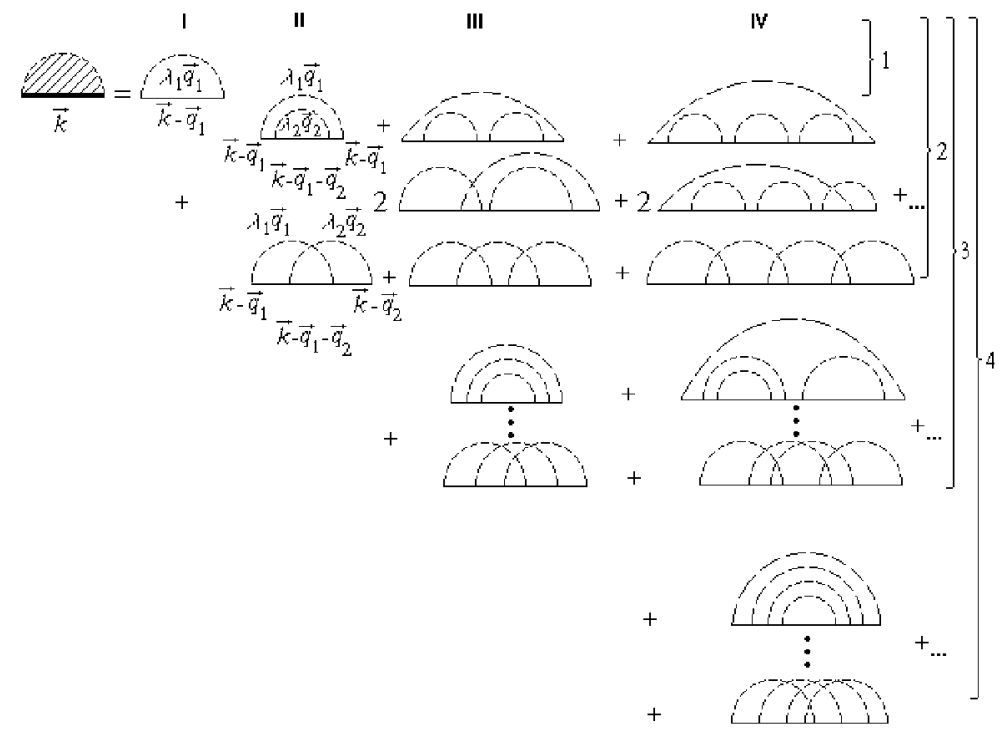

Figure 1.

According to the rules of correspondence between diagrams and analytic expressions in the Pains technique $[5,6]$ (in which the integration over all intermediate frequencies has already been performed) the diagram which takes into account only one-phonon processes gives:

$$
\underbrace{\vec{d}_{1} \vec{q}_{1}}_{\vec{k}-\vec{d}_{1}}=M_{1}^{(1)}\left(\boldsymbol{k}, \omega^{\prime}\right)=\sum_{\lambda_{1} \boldsymbol{q}_{1}} \frac{\left|\varphi_{\lambda_{1}}\left(\boldsymbol{q}_{1}\right)\right|^{2}}{\omega^{\prime}-\varepsilon_{\boldsymbol{k}-\boldsymbol{q}_{1}}-\Omega_{\lambda_{1} \boldsymbol{q}_{1}}} .
$$

The diagrams of class (II) describing two-phonon processes of the first order of the binding function squared module give

$$
\begin{gathered}
=\sum_{\substack{\lambda_{1} \boldsymbol{q}_{1} \\
\lambda_{2} \boldsymbol{q}_{2}}} \frac{\left|\varphi_{\lambda_{1}}\left(\boldsymbol{q}_{1}\right)\right|^{2}\left|\varphi_{\lambda_{2}}\left(\boldsymbol{q}_{2}\right)\right|^{2}}{\left(\omega^{\prime}-\varepsilon_{\boldsymbol{k}-\boldsymbol{q}_{1}}-\Omega_{\lambda_{1} \boldsymbol{q}_{1}}\right)\left(\omega^{\prime}-\varepsilon_{\boldsymbol{k}-\boldsymbol{q}_{1}-\boldsymbol{q}_{2}}-\Omega_{\lambda_{1} \boldsymbol{q}_{1}}-\Omega_{\lambda_{2} \boldsymbol{q}_{2}}\right)} \times \\
\\
{\left[\frac{1}{\omega^{\prime}-\varepsilon_{\boldsymbol{k}-\boldsymbol{q}_{1}}-\Omega_{\lambda_{1} \boldsymbol{q}_{1}}}+\frac{1}{\omega^{\prime}-\varepsilon_{\boldsymbol{k}-\boldsymbol{q}_{2}}-\Omega_{\lambda_{2} \boldsymbol{q}_{2}}}\right] .}
\end{gathered}
$$


Analysing a complete class of diagrams (2), (figure 1) one can be convinced (using an expansion into a geometrical progression series) that a complete infinite set of diagrams accounting one- and two-phonon processes can be obtained as a result of the renormalization of one-phonon MO energy denominator. Consequently, two-phonon $\operatorname{MO} M_{1}^{(2)}\left(\boldsymbol{k}, \omega^{\prime}\right)$ is given by the expression

$$
M_{1}^{(2)}\left(\boldsymbol{k}, \omega^{\prime}\right)=\sum_{\lambda_{1} \boldsymbol{q}_{1}} \frac{\left|\varphi_{\lambda_{1}}\left(\boldsymbol{q}_{1}\right)\right|^{2} A_{1}}{\omega^{\prime}-\varepsilon_{\boldsymbol{k}-\boldsymbol{q}_{1}}-\Omega_{\lambda_{1} \boldsymbol{q}_{1}}-M_{2}^{(1)}\left(\boldsymbol{k}, \omega^{\prime}\right)},
$$

where

$$
\begin{gathered}
M_{2}^{(1)}\left(\boldsymbol{k}, \omega^{\prime}\right)=\sum_{\lambda_{2} \boldsymbol{q}_{2}} \frac{\left|\varphi_{\lambda_{2}}\left(\boldsymbol{q}_{2}\right)\right|^{2} A_{2}\left(\boldsymbol{k}, \omega^{\prime} ; \lambda_{1} \boldsymbol{q}_{1} ; \lambda_{2} \boldsymbol{q}_{2}\right)}{\omega^{\prime}-\varepsilon_{\boldsymbol{k}-\boldsymbol{q}_{1}-\boldsymbol{q}_{2}}-\Omega_{\lambda_{1} \boldsymbol{q}_{1}}-\Omega_{\lambda_{2} \boldsymbol{q}_{2}}} \\
A_{1}=1 ; \quad A_{2}\left(\boldsymbol{k}, \omega^{\prime} ; \lambda_{1} \boldsymbol{q}_{1} ; \lambda_{2} \boldsymbol{q}_{2}\right)=1+\frac{\omega^{\prime}-\varepsilon_{\boldsymbol{k}-\boldsymbol{q}_{1}}-\Omega_{\lambda_{1} \boldsymbol{q}_{1}}}{\omega^{\prime}-\varepsilon_{\boldsymbol{k}-\boldsymbol{q}_{2}}-\Omega_{\lambda_{2} \boldsymbol{q}_{2}}} .
\end{gathered}
$$

The renormalization procedure is universal and true for any order of $(n)$. As the result of its application one can obtain the exact representation of MO in the form of IBIF (since the sum over the wave vectors is considered as the respective integrals)

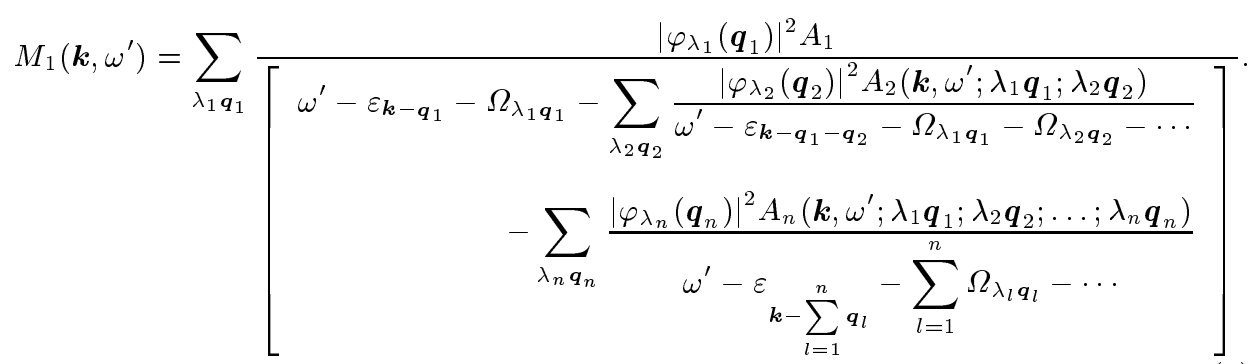

Coefficient $A_{n}$ is given by the expression

$$
\begin{aligned}
& A_{n}\left(\boldsymbol{k}, \omega^{\prime} ; \lambda_{1} \boldsymbol{q}_{1} ; \lambda_{2} \boldsymbol{q}_{2} ; \ldots ; \lambda_{n} \boldsymbol{q}_{n}\right)= \\
& \omega^{\prime}-\varepsilon_{k-\sum_{l=1}^{n-p} \mathbf{q}_{l}}-\sum_{l=1}^{n-p} \Omega_{\lambda_{l} \boldsymbol{q}_{l}}
\end{aligned}
$$

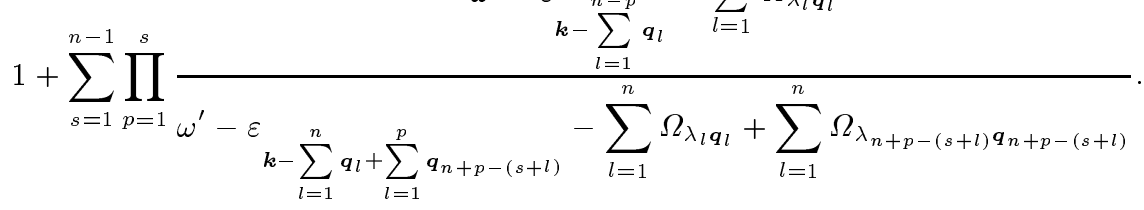

In case when phonon system has only one mode of vibrations $(\lambda=1)$ MO (8) and coefficients $A_{n}(9)$ are equal to the expressions in paper [7] obtained for such model.

The difference in notation is caused by the fact that in paper [7] the abowe result was obtained in the tedious form while expression (9) is compact and convenient for the use. Thus, formulas $(2,8,9)$ are the exact solution of the problem to obtain an analytic expression for the Fourier image of the total GF of the system of quasiparticles interacting with phonons described by the Hamiltonian (1). Herein $G\left(\boldsymbol{k} ; \omega^{\prime}\right)$ is obtained in the form of IBIF with analytically defined branches of the arbitrary $n$-th order. When 
phonon system has one mode $(\lambda=1)$ the IBIF degenerates into the infinite integral continued fraction [7]. The characteristic peculiarity of the representation (8) is the fact that in the branches of the arbitrary $n$-th order the binding functions are in the form $\left|\varphi_{\lambda}(\boldsymbol{q})\right|^{2}$ but not in the powers of arbitrary high orders as it is in the traditional ranges of the Feinman diagram technique.

The topological unity of MO structure (8) allows us to produce an algorithm for its calculation comparatively easily when the dispersion laws of quasiparticles $\left(\varepsilon_{\boldsymbol{k}}\right)$, phonons $\left(\Omega_{\lambda \boldsymbol{q}}\right)$ and binding functions $\left(\varphi_{\lambda}(\boldsymbol{q})\right)$ are fixed. Hence one has to make a truncation of the branching fraction of such order $(n)$ that ensures a necessary exactness when calculating MO. It is clear that the bigger the interaction value $\left|\varphi_{\lambda}(\boldsymbol{q})\right|^{2}$ is the larger the order $(n)$ becomes, which is to be accounted. However as we shall further demonstrate on the example of the concrete model even in the cases when the interaction energy is much bigger than the highest energy of phonon modes, one has to take into account not high number of $n$-phonon processes to obtain the exact result.

In the next section of the present paper the efficiency and usefulness of the mathematical method developed here will be demonstrated to calculate the MO on the example of the system of dispersionless quasiparticles interacting with dispersionless phonons of an arbitrary number of modes. The solution of this problem provides a proof of the exactness of this method since one can compare it with the exact limit result obtained by another independent method. Besides, one can get an upper evaluation of the exactness of the renormalized spectrum of quasiparticles with any dispersion law interacting with phonons having a dispersion.

\section{Spectrum of the system of dispersionless quasiparticles interacting with dispersionless phonons of an arbitrary number of modes}

As an example of the application of the calculating GF Fourier image method developed in the previous paragraph we examine a system of quasiparticles (localized excitons, impurities, etc.) interacting with dispersionless phonons of an arbitrary number of modes (optical vibrations of interbulk and interface modes). Hence $\varepsilon_{\boldsymbol{k}}=\varepsilon, \Omega_{\lambda \boldsymbol{q}}=\Omega_{\lambda}$ and since the sum over $\boldsymbol{q}$ in $\mathrm{MO}(8)$ is now related to the binding functions only; it is convenient to introduce term $\Phi_{\lambda}^{2}=\sum_{\boldsymbol{q}}\left|\varphi_{\lambda}(\boldsymbol{q})\right|^{2}$ and dimensionless parameter $\alpha_{\lambda}=\Phi_{\lambda} / \Omega_{\lambda}$ characterizing the magnitude of the energy of the quasiparticle interaction with phonons of $\lambda$-th mode in the units of phonon energy of this mode.

Although for such a model the exact integral representation of the GF Fourier image is known [5]

$$
G\left(\omega^{\prime}\right)=\frac{\exp \left(-\sum_{\lambda} \alpha_{\lambda}^{2}\right)}{2 \pi \mathrm{i}} \int_{0}^{\infty} \mathrm{d} t \exp \left\{\mathrm{i}\left(\omega^{\prime}-\varepsilon-\sum_{\lambda=1}^{\tau} \alpha_{\lambda}^{2} \Omega_{\lambda}\right) t+\sum_{\lambda=1}^{\tau} \alpha_{\lambda}^{2} \exp \left(-\mathrm{i} \Omega_{\lambda} t\right)\right\}
$$

it is impossible to obtain the exact spectrum of the system using it directly. When operator of the number of quasiparticles is equal to that squared one can perform a canonical transformation [1] defining the renormalized energy 
spectrum of the system:

$$
\varepsilon_{\lambda_{1}, \ldots, \lambda_{n}}^{(n)}=\varepsilon-\sum_{\lambda=1}^{\tau} \alpha_{\lambda}^{2} \Omega_{\lambda}+\sum_{l=1}^{n} \Omega_{\lambda_{l}}, \quad(n=0,1,2, \ldots, \infty) .
$$

It is clear that this spectrum contains the basic state corresponding to the existence in the system of the quasiparticle with the renormalized energy $E^{(0)}=E-\sum_{\lambda=1}^{\tau} \alpha_{\lambda}^{2} \Omega_{\lambda}$ and an infinite number of excited states of the system corresponding to the energies of the system in which a quasiparticle exists with one of the phonons of $\lambda$-th mode $\left(E_{\lambda}^{1}=E^{(0)}+\Omega_{\lambda}\right)$, with two phonons $\left(E_{\lambda_{1} \lambda_{2}}^{2}=E^{(0)}+\Omega_{\lambda_{1}}+\Omega_{\lambda_{2}}\right)$, etc.

The spectrum of the considered system without any additional restrictions is obtained with the help of the IBIF method. In this case the branching integral fraction for the MO (8) degenerates into a simple branching fraction (unintegral) since the sum over $\boldsymbol{q}$ is related to the binding functions and not to the energies of quasiparticles and phonons. Introducing convenient dimensionless phonon energies $\tilde{\Omega}_{\lambda}=\Omega_{\lambda} / \Omega$, frequency $\xi=(\omega-E) / \Omega$, one can get from (8) the total MO $\mathfrak{M}(\xi)=M_{1}(\omega) / \Omega$ expressed in the units of minimal phonon energies

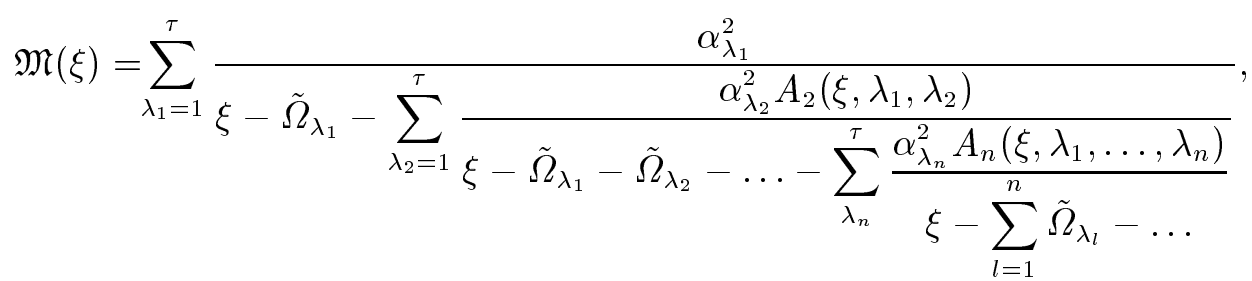

where

$$
A_{n}\left(\xi, \lambda_{1}, \ldots, \lambda_{n}\right)=1+\sum_{s=1}^{n-1} \prod_{p=1}^{s} \frac{\xi-\sum_{l=1}^{n-p} \tilde{\Omega}_{\lambda_{l}}}{\xi-\sum_{l=1}^{n} \tilde{\Omega}_{\lambda_{l}}+\sum_{l=1}^{p} \tilde{\Omega}_{\lambda_{n+p-(s+l)}}}
$$

The dispersion equation

$$
\xi=\mathfrak{M}(\xi)
$$

defines the energy spectrum of the system.

It is clear that when phonon system has one mode of vibrations $(\lambda=1)$ since $A_{n}=n, \alpha_{1}=\alpha$ and infinite branching fraction (12) degenerates into an infinite continuous fraction

$$
\mathfrak{M}(\xi)=\frac{\alpha^{2}}{\xi-\frac{2 \alpha^{2}}{\xi-2-\ldots-\frac{n \alpha^{2}}{\xi-n-\ldots}}},
$$

it was analysed in details in the paper [7].

The monotype structure of the branches of all orders of MO in the form (12) gives opportunity to obtain the spectrum solving the dispersion 
equation (14). Analysing $\mathfrak{M}(\xi)$ it is necessary to pay attention to the principal difference of its frequency dependence in case of one-mode and twoor many-mode phonon systems. In figures 2 and 3 the typical examples of the dependences of the quasiparticle MO for the cases of its interaction with one-mode (figure 2) $\tilde{\Omega}=1, \alpha=0.8$ and two-mode (figure 3 ) $\tilde{\Omega}_{a}=1$, $\alpha_{a}=0.3, \tilde{\Omega}_{b}=1.6, \alpha_{b}=0.2$ phonon systems are presented. Figures 2 and 3 show that irrespectively of the number of modes $(\tau)$ with which quasiparticles interact in the region $\xi \approx-\sum_{\lambda} \alpha_{\lambda}^{2} \tilde{\Omega}_{\lambda}$, MO (12) is a smooth function of frequency. Therefore, the dispersion equation (14) has the only solution

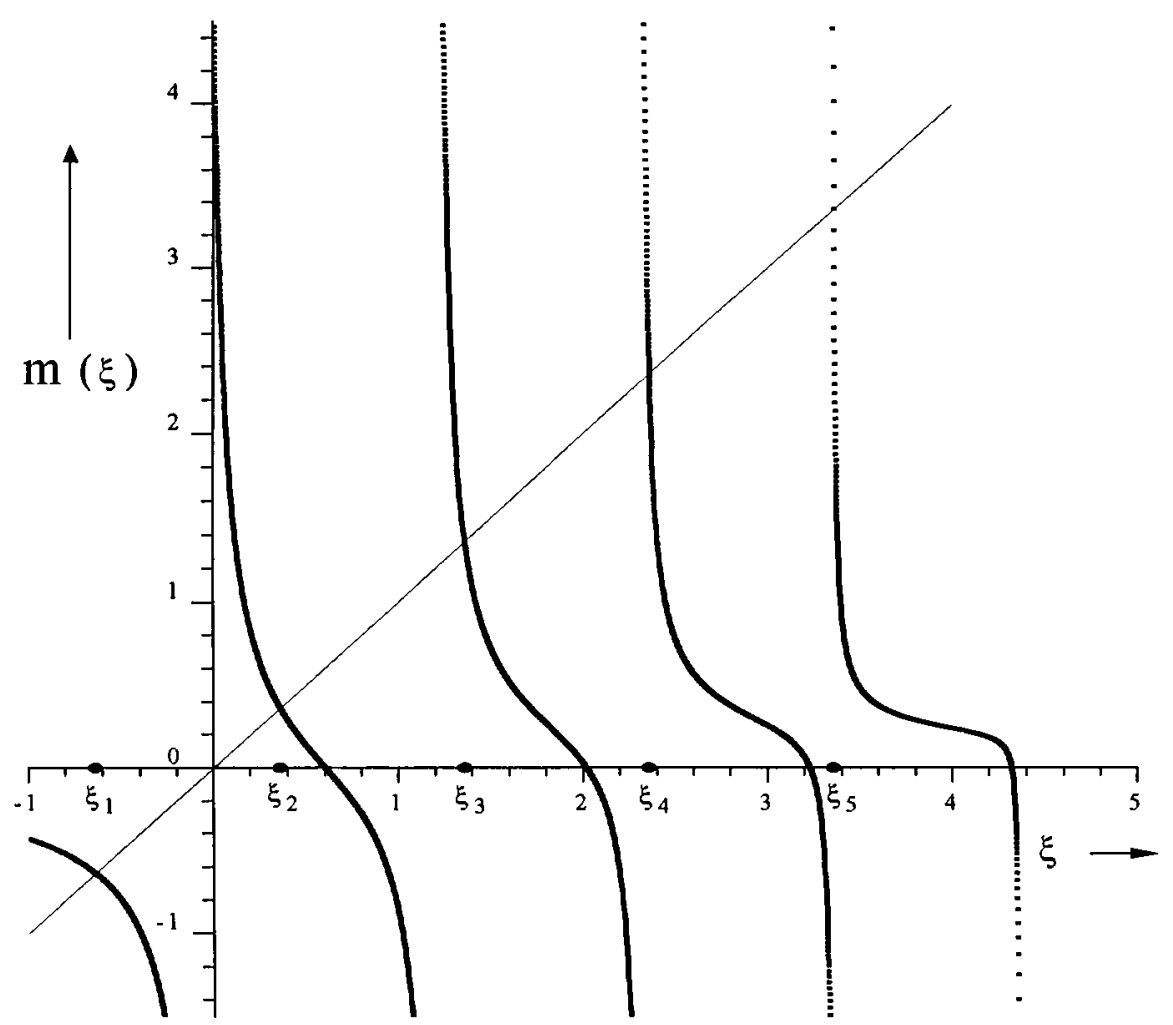

Figure 2 .

here. It will be shown further that this solution at big $n$ values is the same as the result [1] of the canonical transformation with an additional condition on the operator of the number of quasiparticles. It defines the renormalized energy of the basic state

$$
\xi_{0}=-\sum_{\lambda} \alpha_{\lambda}^{2} \tilde{\Omega}_{\lambda}
$$

In the region of the excited states the frequency dependence of MO of quasiparticles interacting with one-, two-, or many-mode phonon systems is fairly different. In case of a one-mode phonon system (figure 2) between two arbitrary neighbouring energies of excited states the quasiparticles MO as a function of frequency has one rupture. Therefore, the dispersion equation (14) defines the energies of all excited states in accordance to the results 


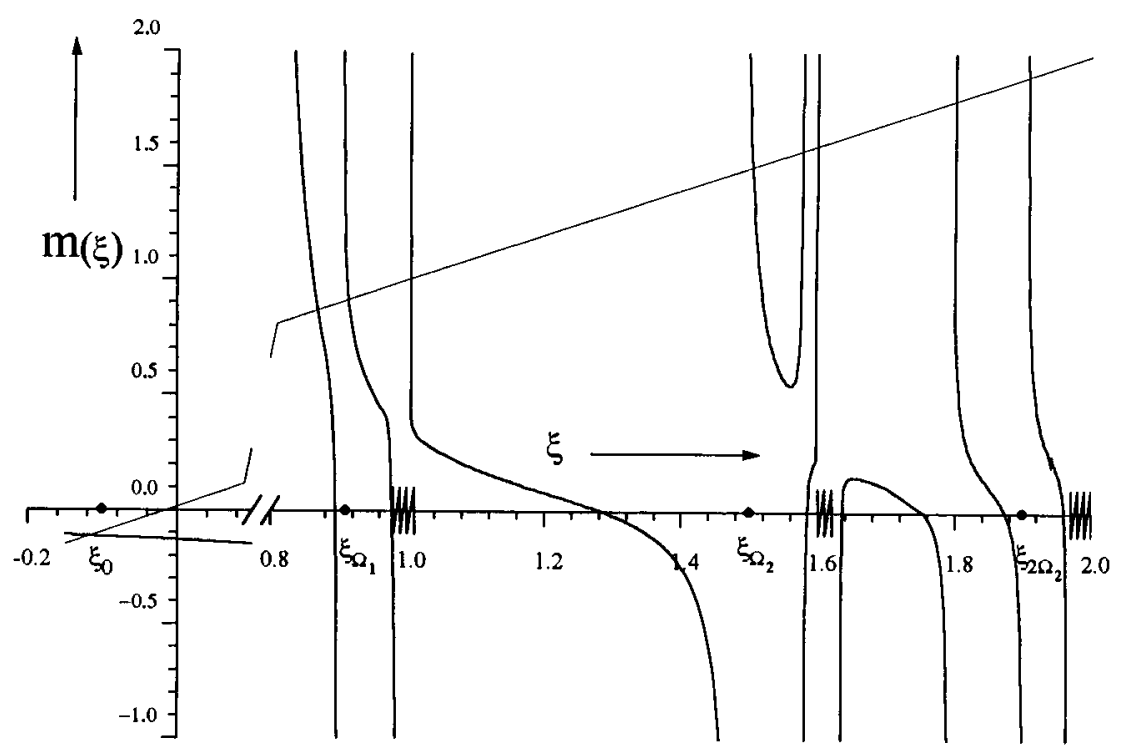

Figure 3.

presented in the paper [1]

$$
\xi_{n}=-\sum_{\lambda} \alpha_{\lambda}^{2} \tilde{\Omega}_{\lambda}+n
$$

In case of two-mode (figure 3) or many-mode systems in all frequency regions between any two neighbouring combinations of phonon energies MO has not one but an infinite number of ruptures the distance between which vanishes when the frequency increases. It means that in the region of all possible frequences of the excited system there exists not one state with the energies defined by formula (17) but indeed in every region there is an infinite set of energy states creating a series converging into the region of higher energies (figure 3). The situation described here is clear from the physical considerations. Indeed, the interaction between the quasiparticles and phonons of different modes is equivalent to the interaction of the phonons of different modes with each other through a system of quasiparticles. Naturally, such interaction causes the cardinal rebuilding of the phonon spectrum and thus creates a complicated spectrum of the excited states of the system. Neglecting the difference between the operator of the number of particles and its square value to realize the canonical transformation brings to the fictitious degeneration of the infinite series of the system excited states. Since the basic renormalized state of the system corresponding to the energy of the quasiparticle is not degenerated (according to the quantum mechanics principles) thus the values of this energy are equal in the both methods of calculation. The total spectrum of the system is also equal in the both methods due to the absence of intermode interaction in one-mode phonon model (it is shown in the figure by means of the vertical dashed lines in the corresponding regions of the variable $\xi$ ).

In figure 4 (parts a, b, c) the results of the calculation of the renormalized values of the basic state energies $\left(\xi_{0}^{(n)}\right)$ of the systems with a different number of modes depending on the number of branches $(n)$ accounted in MO are 
presented. Figure 4a corresponds to the one-mode system $\left(\tilde{\Omega}_{1}=1\right)$, figure $4 \mathrm{~b}$ - to the two-mode system $\left(\tilde{\Omega}_{1}=1, \tilde{\Omega}_{2}=1.6\right)$, figure $4 \mathrm{c}-$ to the three mode system $\left(\tilde{\Omega}_{1}=1, \tilde{\Omega}_{2}=1.6,\left(\tilde{\Omega}_{3}=2.1\right)\right.$. Magnitudes $\alpha_{s} / \tilde{\Omega}_{s}$ are marked by the numerals above the respective curves in the figures with the assumption: $\alpha_{1} / \tilde{\Omega}_{1}=\alpha_{2} / \tilde{\Omega}_{2}=\alpha_{3} / \tilde{\Omega}_{3}$. Horizontal lines to the right of all three figures correspond to the exact values (16) of the renormalized basic state energies of the system defined by the canonical transformation.
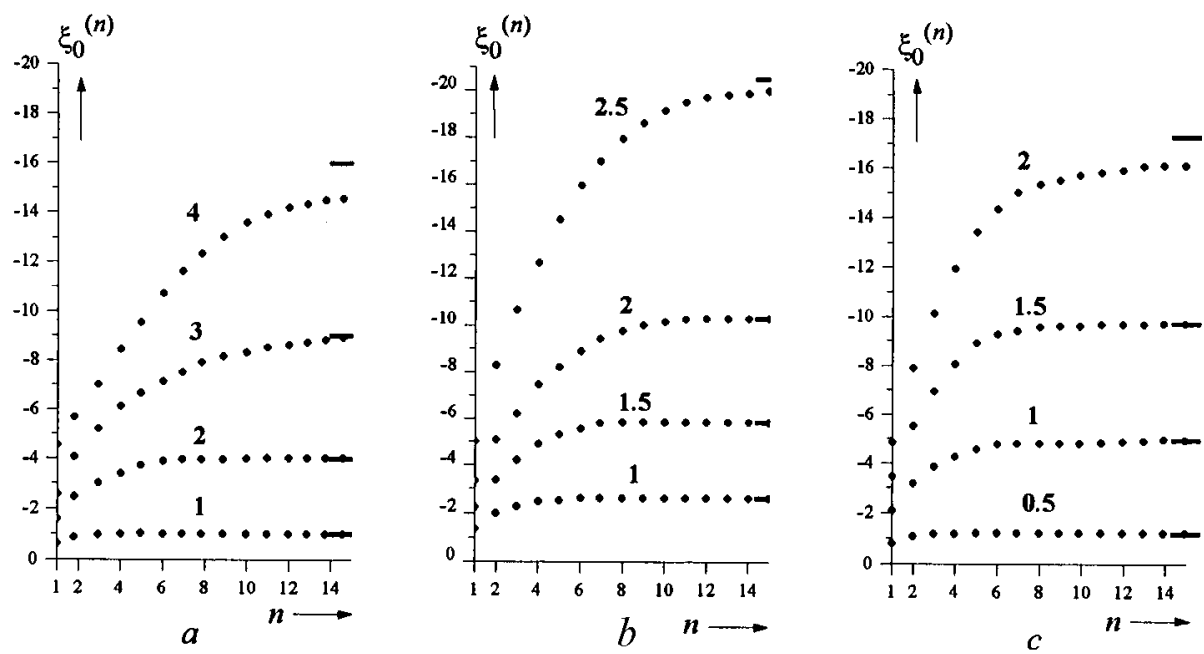

Figure 4.

From figure 4 one can see that in case of weak binding when the interaction energy is less than the energy of the respective phonon $\left(\alpha_{s}<1\right)$ the accounting of some first groups of branches in MO gives rather an exact result for the energy values of the renormalized basic states $\left(\xi_{0}\right)$. With an increase of the number of system modes and interaction value the fixed exactness of the calculated value is reached by means of the increasing number of the accounted groups of branches in MO. However, it is clear that this value is comparably small even when the interaction energy is 2-4 times bigger than the energy of respective phonon modes.

The calculation of $\xi_{0}$ in the one-mode model showed that in the case of tight binding when e.g. the interaction energy is one order bigger than phonon energy $(\alpha=10)$, the renormalized value of the basic state energy at $n=25$ is $\xi_{0}^{(n=25)}=-9.99983$. It differs from the exact $\xi_{0}=-10$ less than by $0.002 \%$. Thus one can make a conclusion that taking account the possibilities of computers the IBIF method provides the effective way to calculate the spectrum of the system of quasiparticles interacting with phonons at arbitrary binding values. It does not cause the non-physical discrepancies in the higher orders of the theory (what is inherent to the traditional diagram technique approach [4-6]). On the contrary, taking into account the groups of branches of higher orders regularly approximates the exact spectrum more precisely. Mathematical reasons for such a positive situation are the fact that in the approach developed in the present paper the expansion in power series of binding functions is not present in an evident form with which as Pains mentioned correctly in [6] "nature does not agree". 


\title{
References
}

[1] Davydov A.S. Solid state theory. Moscow, Nauka, 1976.

[2] Mori N. Transport, collective motion and Brounian motion. // Progr. Theor. Phys., 1965, vol. 33, p. 423-428.

[3] Tserkovnikov Yu. About the method of the solving of infinite systems of equatios for the two-time temperature Green functions. // Teor. Mat. Fiz., 1981, vol. 49, No 2, p. 219-233 (in Russian).

[4] Abrikosov A.A., Gorkov L.P., Dzjaloshinsky J.M. Methods of quantum field theory in statistical physics. Moscow, Fizmatgiz, 1962.

[5] Agranovich V.M. Theory of excitons. Moscow, Nauka, 1968.

[6] Pines D. The many-body problem. New-York, W.A.Benjamin Inc., 1961.

[7] Tkach N.V. Method of the system of exact equations for the irreducible selfenergy of quasiparticles interacting with phonons. // Teor. Mat. Fiz., 1984, vol. 61, No 3, p. 400-407 (in Russian).

[8] Tkach N.V., Lopyshanskaja N.V., Val A.D. About the one method of the effective accounting of many-phonon processes in the problems of the spinless quasiparticles interacting with the lattice vibrations at the finite temperatures. // Izv. Vuzov., Fiz., 1986, vol. 10, p. 93-98 (in Russian).

[9] Sherman A.V. Exciton absorption spectrum (I). // Phys. Stat. Sol. (b), 1985, vol. 131, p. 225-231.

[10] Sherman A.V. Exciton absorbtion spectrum (II). // Phys. Stat. Sol. (b), 1986, vol. 135 , No 2, p. 697-704.

[11] Val A.D., Tkach N.V. Tre renormalization of the spectrum of two-dimensional exciton due to the manyphonon processes in magnetic field. // Izv. Vuzov, Fiz., 1987, vol. 4, No 4, p. 3-7 (in Russian).

[12] Tkach N.V., Zharkoi V.P. Cascade peculiarities in the spectrum of quasiparticles interacting with phonons. // Izv. Vuzov, Fiz., 1990, vol. 4, No 11, p. 17-20 (in Russian).

[13] Tkach N.V., Val A.D., Zharkoi V.P. The spectra of diamagnetic and WannierMott excitons in ionic crystals in the region of energies of the fundamental peak and two phonon replicas. // Fiz. Tverd. Tela., 1992, vol. 34, No 11, p. 3543-3548 (in Russian).

\section{МЕТОД БЕЗМЕЖКНОГО РОЗГАЛУЖЕНОГО ІНТЕГРАЛЬНОГО ДРОБУ В ТЕОРІЇ ФУНКЦІЙ ГРІНА}

\author{
М.В.Ткач
}

Для системи квазічастинок, що взаємодіють з фононами довільної кількості віток при $T=0 \mathrm{~K}$, на основі діаграмної техніки Фейнмана знайдено точне представлення функції Гріна у вигляді безмежного розгалуженого інтегрального дробу. Показано, що оскільки розвинутий метод однотипно враховує багатофононні процеси, то він дозволяє швидко й ефективно знаходити перенормований взаємодією спектр системи при довільних величинах зв'язку і у довільних областях енергій та квазііпульсів. Показано, що у системі бездисперсійних квазічасток, що взаємодіють з багатомодовими фононами, збуджені стани утворюють безмежні збіжні серії енергетичних рівнів. 